\title{
On Common Fixed Point Theorem in Complete metric Space
}

\author{
${ }^{1}$ Mrs. Jyoti Gupta, ${ }^{2}$ Dr. P. L. Sanodia , ${ }^{3}$ Dr.K.Qureshi Add. Director (Rtd), \\ ${ }^{4}$ Mrs. Anupama Gupta \\ ${ }^{1}$ Institute for Excellence in higher Education Bhopal (India)
}

\begin{abstract}
We prove a unique common fixed-point theorem for two pair of weakly compatible maps in a complete metric space, which generalizes the result of Brian Fisher by a weaker condition such as weakly compatibility instead of compatibility and contractive modulus instead of continuity of maps.
\end{abstract}

Keywords: Fixed point, Common fixed point, Contractive modulus, weakly compatible maps, and complete metric space.

\section{Introduction}

The concept of the commutativity has generalized in several ways. For this Sessa, S. [8] has introduced the concept of weakly commuting and Jungck, G. [4] initiated the concept of compatibility. It can be easily verified that when the two mappings are commuting then they are compatible but not conversely.In1998, Jungck and Rhoades [5] introduced the notion of weakly compatible and showed that compatible maps are weakly compatible but Not conversely. Brain Fisher [2] proved an important Common Fixed Point theorem.

The study of common fixed point of mapping satisfying contractive type conditions has been a very active field of research activity during the last three decades. Jungck [4] introduced more generalized commuting mappings, called compatible mappings, which are more general than commuting and weakly commuting mapping.

The main purpose of this paper is to present fixed point results for two pair of maps satisfying a new contractive condition by using the concept of weakly compatible maps in a complete metric space. We recall the definitions of complete metric space, the notion of convergence and other results that will be needed in the sequel.

Definition 2.1 Let $\mathrm{f}$ and $\mathrm{g}$ be two self-maps on a set $\mathrm{X}$. If $\mathrm{fx}=\mathrm{gx}$, for some $\mathrm{x}$ in $\mathrm{X}$ then $\mathrm{x}$ is called coincidence point of $f$ and $g$.

Definition 2.2 Let $f$ and $g$ be two self-maps defined on a set, then $f$ and $g$ are said to be weakly compatible if they commute at coincidence points. That is, if $f u=g u$ for some $\mathrm{u} \in \mathrm{X}$, then fgu $=\mathrm{gfu}$.

Definition 2.3 Let $f$ and $g$ be weakly compatible self mapping of a set $X$. if $f$ and $g$ have a unique point of coincidence, that is $\mathrm{w}=\mathrm{fx}=\mathrm{gx}$, then $\mathrm{w}$ is the unique common fixed point of $\mathrm{f}$ and $\mathrm{g}$.

Definition 2.4 A function $\emptyset:[0, \infty) \rightarrow[0, \infty)$ is said to be a contractive modulus if $[0, \infty)$ and $\emptyset(t)<t$ for $\mathrm{t}>0$

$\emptyset:[0, \infty) \rightarrow$

Definition 2.5 A real valued function $\emptyset$ defined on $X \subseteq \mathrm{R}$ is said to be upper semi continuous. If $\lim _{n \rightarrow \infty} \sup \emptyset\left(t_{n}\right) \leq \emptyset(t)$, for every sequence $\left\{t_{n}\right\} \in X$ with $t_{n} \rightarrow t$ as $n \rightarrow \infty$.It is clear that every continuous function is upper semi continuous but converse may not true.

\section{Main Result}

Theorem 3.1 Suppose S, P, T and Q are four self maps of a metric space (X, d) satisfying the following conditions,

(1) $\mathrm{S}(\mathrm{X}) \subseteq \mathrm{Q}(\mathrm{X})$ and $\mathrm{T}(\mathrm{X}) \subseteq \mathrm{P}(\mathrm{X})$.

(2) Pairs (S, P) and (T, Q) are commuting.

(3) One of S, P, T and Q is continuous.

(4) $\mathrm{d}(\mathrm{Sx}, \mathrm{Ty}) \leq c(\lambda(\mathrm{x}, \mathrm{y}))$.

where $\lambda(x, y)=\max \{d(P x, Q y), d(P x, S x), d(Q y, T y), d(P x, T y), d(Q y, S x)\}$

For all $\mathrm{x}, \mathrm{y} \in X$ and $0 \leq c<1$.

Further if

(5) $\mathrm{X}$ is complete.

Then $\mathrm{S}, \mathrm{P} \mathrm{T}$ and $\mathrm{Q}$ have a unique common fixed point $\mathrm{z} \mathrm{EX}$. Also $\mathrm{z}$ is the unique common fixed point of $(\mathrm{S}, \mathrm{P})$ and of $(\mathrm{T}, \mathrm{Q})$.

Proof: Suppose $x_{0}$ is an arbitrary point of $\mathrm{X}$ and define the sequence $\left\{y_{n}\right\}$ is $\mathrm{X}$ such that

By (ii) we have,

$$
\begin{gathered}
y_{n}=\mathrm{S} x_{n}=\mathrm{Q} x_{n+1} \\
y_{n+1}=\mathrm{T} x_{n+1}=\mathrm{P} x_{n+2}
\end{gathered}
$$




$$
\begin{aligned}
\mathrm{d}\left(y_{n}, \mathrm{y}_{\mathrm{n}+1}\right)= & \mathrm{d}\left(\mathrm{S} x_{n}, \mathrm{~T} x_{n+1}\right) \\
& \leq \mathrm{c}\left(\lambda\left(x_{n}, x_{n+1}\right)\right)
\end{aligned}
$$

Where

$$
\begin{aligned}
\lambda\left(x_{n}, x_{n+1}\right)= & \max \left\{\mathrm{d}\left(\mathrm{P} x_{n}, \mathrm{Q} x_{n+1}\right), \mathrm{d}\left(\mathrm{P} x_{n}, \mathrm{~S} x_{n}\right), \mathrm{d}\left(\mathrm{Q} x_{n+1}, \mathrm{~T} x_{n+1}\right),\right. \\
& \left.\mathrm{d}\left(\mathrm{P} x_{n}, \mathrm{~T} x_{n+1}\right), \mathrm{d}\left(\mathrm{Q} x_{n+1}, \mathrm{~S} x_{n}\right)\right\} \\
= & \max \left\{\mathrm{d}\left(\mathrm{T} x_{n-1}, \mathrm{~S} x_{n}\right), \mathrm{d}\left(\mathrm{T} x_{n-1}, \mathrm{~S} x_{n}\right), \mathrm{d}\left(\mathrm{S} x_{n}, \mathrm{~T} x_{n+1}\right),\right. \\
& \left.\quad \mathrm{d}\left(\mathrm{T} x_{n-1}, \mathrm{~T} x_{n+1}\right), \mathrm{d}\left(\mathrm{S} x_{n}, \mathrm{~S} x_{n}\right)\right\} \\
= & \max \left\{\left(d\left(y_{n-1}, y_{n}\right), d\left(y_{n-1}, y_{n}\right), \mathrm{d}\left(y_{n}, y_{n+1}\right), \mathrm{d}\left(y_{n-1}, y_{n+1}\right), d\left(y_{n}, y_{n}\right)\right\}\right. \\
= & \max \left\{\left(d\left(y_{n-1}, y_{n}\right), \mathrm{d}\left(y_{n}, y_{n+1}\right), \mathrm{d}\left(y_{n-1}, y_{n}\right), d\left(y_{n}, y_{n+1}\right), 0\right\}\right. \\
\leq & \max \left\{\left(d\left(y_{n-1}, y_{n}\right), \mathrm{d}\left(y_{n}, y_{n+1}\right)\right\}\right.
\end{aligned}
$$

Since $\mathrm{c}$ is a contractive modulus, $\lambda\left(x_{n}, x_{n+1}\right)=\mathrm{d}\left(y_{n}, y_{n+1}\right)$ is not possible.

Thus

$$
\mathrm{d}\left(y_{n}, y_{n+1}\right) \leq \mathrm{c}\left(d\left(y_{n-1}, y_{n}\right)\right)
$$

Since c an upper semi continuous, contractive modulus equation (1) implies that the sequence $\mathrm{d}\left(y_{n+1}, y_{n}\right)$ is monotonic decreasing and continuous.

Hence there exists a real number, say $r \geq 0$

Such that $\lim _{n \rightarrow \infty} \mathrm{d}\left(y_{n+1}, y_{n}\right)=\mathrm{r}$

Therefore as $\mathrm{n} \rightarrow \infty$ equation (1) implies that $\mathrm{r} \leq \mathrm{c}(\mathrm{r})$

Which is possible only if $\mathrm{r}=0$ because $\mathrm{c}$ is a contractve modulus.

Thus $\lim _{n \rightarrow \infty} d\left(y_{n+1}, y_{n}\right)=0$

Now we show that $\left\{y_{n}\right\}$ is a Cauchy sequence

Let if possible we assume that $\left\{y_{n}\right\}$ is not a Cauchy sequence.

Then there exists an $\varepsilon>0$ and subsequence $\left\{n_{i}\right\}$ and $\left\{m_{i}\right\}$ such that

$m_{i}<n_{i}<m_{i+1}$ and

$d\left(y_{m_{i}}, y_{n_{i}}\right) \geq \varepsilon$ and $d\left(y_{m_{i}}, y_{n_{i}-1}\right)<\varepsilon \ldots(2)$

So that $\varepsilon \leq d\left(y_{m_{i}}, y_{n_{i}}\right) \leq d\left(y_{m_{i}}, y_{n_{i}-1}\right)+d\left(y_{n_{i}-1}, y_{n_{i}}\right)<\varepsilon+\left(y_{n_{i}-1}, y_{n_{i}}\right)$

Therefore $\lim _{i \rightarrow \infty} d\left(y_{m_{i}}, y_{n_{i}}\right)=\varepsilon$

Now $d\left(y_{m_{i}-1}, y_{n i-1}\right) \leq d\left(y_{m_{i}-1}, y_{m_{i}}\right)+d\left(y_{m_{i}}, y_{n_{i}}\right)+d\left(y_{n_{i}}, y_{n_{i}-1}\right)$

By taking limit as $\mathrm{i} \rightarrow \infty$ we get $\lim _{i \rightarrow \infty} d\left(y_{m_{i}-1}, y_{n_{i}-1}\right)=\varepsilon$

Now by (ii) and (2)

$$
\begin{gathered}
\varepsilon \leq d\left(y_{m_{i}}, y_{n_{i}}\right)=d\left(S x_{m_{i}}, T x_{n_{i}}\right) \leq c\left(\lambda\left(x_{m_{i}}, x_{n_{i}}\right)\right) \\
\text { i.e., } \varepsilon \leq c\left(\lambda\left(y_{m_{i}}, y_{n_{i}}\right)\right) \ldots 3 \\
x=x_{m_{i}}, y=x_{n_{i}} \\
\lambda\left(x_{m_{i}}, x_{n_{i}}\right)=\max \left\{d\left(P x_{m_{i}}, Q x_{n_{i}}\right)\left(P x_{m_{i}}, S x_{m_{i}}\right), d\left(Q x_{n_{i}}, T x_{n_{i}}\right),\right. \\
\left.d\left(P x_{m_{i}}, T x_{n_{i}}\right), d\left(Q x_{n_{i}}, S x_{m_{i}}\right)\right\} \\
=\max \left\{d\left(T x_{m_{i}-1}, S x_{n_{i}-1}\right), d\left(T x_{m_{i}-1}, S x_{m_{i}}\right), d\left(S x_{n_{i}-1}, T x_{n_{i}}\right),\right. \\
\left.d\left(T x_{m_{i}-1}, T x_{n_{i}}\right), d\left(S x_{n_{i}-1}, y_{m_{i}}\right)\right\} \\
=\max \left\{d\left(y_{m_{i}-1}, y_{n_{i}-1}\right), d\left(y_{m_{i}-1}, y_{m_{i}}\right), d\left(y_{n_{i}-1}, y_{n i}\right),\right. \\
\left.d\left(y_{m_{i}-1}, y_{n_{i}}\right), d\left(y_{n i-1}, y_{m_{i}}\right)\right\}
\end{gathered}
$$

By taking limit as $\mathrm{i} \rightarrow \infty$, we get

$$
\operatorname{Lim}_{i \rightarrow \infty} \lambda\left(y_{m_{i}}, y_{n_{i}}\right)=\max \{\varepsilon, 0,0, \varepsilon, \varepsilon\}
$$

Thus we have, $\operatorname{Lim}_{i \rightarrow \infty} \lambda\left(x_{m_{i}}, x_{n_{i}}\right)=\varepsilon$

Therefore from (3) $\quad \varepsilon \leq \varphi(\varepsilon)$

This is a contradiction because $0<\varepsilon$ and $\varphi$ is contractive modulus.

Thus $\left\{y_{n}\right\}$ is a Cauchy sequence in $\mathrm{X}$.

Since $\mathrm{X}$ is complete, there exists a point $\mathrm{z}$ in $\mathrm{X}$ such that

$\operatorname{Lim}_{n \rightarrow \infty} y_{n}=z$

Thus $\operatorname{Lim}_{n \rightarrow \infty} S x_{n}=\operatorname{Lim}_{n \rightarrow \infty} Q x_{n+1}=z$ and $\operatorname{Lim}_{n \rightarrow \infty} T x_{n+1}=\operatorname{Lim}_{n \rightarrow \infty} P x_{n+2}=z$

$$
\text { i.e., } \operatorname{Lim}_{n \rightarrow \infty} S x_{n}=\operatorname{Lim}_{n \rightarrow \infty} Q x_{n+1}=\operatorname{Lim}_{n \rightarrow \infty} T x_{n+1}=\operatorname{Lim}_{n \rightarrow \infty} P x_{n+2}=z
$$

since $\mathrm{T}(\mathrm{X}) \subseteq \mathrm{P}(\mathrm{X})$. There exists a point $\mathrm{u} \in \mathrm{X}$

such that $\mathrm{z}=\mathrm{Pu}$

Then by (ii), we have 
$\mathrm{d}(\mathrm{Su}, \mathrm{z}) \leq \mathrm{d}\left(\mathrm{Su}, \mathrm{T} x_{n+1}\right)+\mathrm{d}\left(\mathrm{T} x_{n+1}, z\right)$

$\leq \mathrm{c}\left(\lambda\left(\mathrm{u}, x_{n+1}\right)\right)+\mathrm{d}\left(\mathrm{T} x_{n+1}, \mathrm{z}\right)$

Where $\left.\lambda\left(\mathrm{u}, x_{n+1}\right)\right)=\max \left\{d\left(P u, Q x_{n+1}\right), d(P u, S u), d\left(Q x_{n+1}, T x_{n+1}\right)\right.$, $\left.d\left(P u, T x_{n+1}\right), d\left(Q x_{n+1}, S u\right)\right\}$

Taking the limit as $\mathrm{n} \rightarrow \infty$

$$
=\max \left\{d\left(z, S x_{n}\right), d(z, S u), d\left(S x_{n}, T x_{n+1}\right), d\left(z, T x_{n+1}\right), d\left(S x_{n}, S u\right)\right\}
$$

$\lambda\left(\mathrm{u}, x_{n+1}\right)=\max \{d(z, z), d(z, S u), d(z, z), d(z, z), d(z, S u)\}$

$$
=\mathrm{d}(\mathrm{z}, \mathrm{Su})
$$

Thus as $\mathrm{n} \rightarrow \infty$

$\mathrm{d}(\mathrm{Su}, \mathrm{z}) \leq \mathrm{c}(\mathrm{d}(\mathrm{Su}, \mathrm{z}))+\mathrm{d}(\mathrm{z}, \mathrm{z})=\mathrm{c}(\mathrm{d}(\mathrm{Su}, \mathrm{z}))$

If $\mathrm{Su} \neq \mathrm{z}$ then $\mathrm{d}(\mathrm{Su}, \mathrm{z})>0$ and hence as $\mathrm{c}$ is contractive modulus

$\mathrm{c}(\mathrm{d}(\mathrm{Su}, \mathrm{z}))<\mathrm{d}(\mathrm{Su}, \mathrm{z})$.

Therefore $\mathrm{d}(\mathrm{Su}, \mathrm{z})<\mathrm{d}(\mathrm{Su}, \mathrm{z})$, Which is a contradiction.

Thus $\mathrm{Su}=\mathrm{z}$. $\mathrm{So} \mathrm{Pu}=\mathrm{Su}=\mathrm{z}$

So $u$ is a coincidence point of $P$ and $S$

Since the pair of map $\mathrm{S}$ and $\mathrm{P}$ are weakly compatible, $\mathrm{SPu}=\mathrm{PSu}$.

\section{$\mathrm{Sz}=\mathrm{Pz}$}

Again Since $S(X) \subseteq Q(X)$, there exist a point $v \in X$

Then by (ii), we have

$\mathrm{d}(\mathrm{z}, \mathrm{Tv})=\mathrm{d}(\mathrm{Su}, \mathrm{Tv}) \leq \mathrm{c}(\lambda(\mathrm{u}, \mathrm{v}))$

where

$\lambda(u, v)=\max \{d(P u, Q u), d(P u, S u), d(Q v, T v), d(P u, T v), d(Q v, S u)\}$

$=\max \{\mathrm{d}(\mathrm{z}, \mathrm{z}), \mathrm{d}(\mathrm{z}, \mathrm{z}), \mathrm{d}(\mathrm{z}, \mathrm{Tv}), \mathrm{d}(\mathrm{z}, \mathrm{Tv}), \mathrm{d}(\mathrm{z}, \mathrm{z})\}$

$=\max \{0,0, \mathrm{~d}(\mathrm{z}, \mathrm{Tv}), \mathrm{d}(\mathrm{z}, \mathrm{Tv}), 0\}$

$=\mathrm{d}(\mathrm{z}, \mathrm{Tv})$

Thus $\mathrm{d}(\mathrm{z}, \mathrm{Tv}) \leq \mathrm{c}(\mathrm{d}(\mathrm{z}, \mathrm{Tv}))$

If $\mathrm{Tv} \neq \mathrm{z}$ and $\mathrm{d}(\mathrm{z}, \mathrm{Tv})>0$ and hence as $\mathrm{c}$ is contractive modulus $\mathrm{c}(\mathrm{d}(\mathrm{z}, \mathrm{Tv}))<\mathrm{d}(\mathrm{z}, \mathrm{Tv})$

Therefore $\mathrm{d}(\mathrm{z}, \mathrm{Tv})<\mathrm{d}(\mathrm{z}, \mathrm{Tv})$ which is a contradiction .Therefore $\mathrm{Tv}=\mathrm{Qv}=\mathrm{z}$, so $\mathrm{v}$ is a coincidence point of $\mathrm{Q}$ and $T$. Since the pair of maps $Q$ and $T$ are weakly compatible, $Q T v=T Q v$, i. e., Qz $=T z$.

Now we show that $\mathrm{z}$ is a fixed point of $\mathrm{S}$

By (ii) we have

$\mathrm{d}(\mathrm{Sz}, \mathrm{z})=\mathrm{d}(\mathrm{Sz}, \mathrm{Tv})$

$\leq \mathrm{c}(\lambda(\mathrm{z}, \mathrm{v}))$

where

$\lambda(\mathrm{z}, \mathrm{v})=\max \{\mathrm{d}(\mathrm{Pz}, \mathrm{Qv}), \mathrm{d}(\mathrm{Pz}, \mathrm{Sz}), \mathrm{d}(\mathrm{Qv}, \mathrm{Tv}), \mathrm{d}(\mathrm{Pz}, \mathrm{Tv}), \mathrm{d}(\mathrm{Qv}, \mathrm{Sz})\}$

$=\max \{\mathrm{d}(\mathrm{Sz}, \mathrm{z}), \mathrm{d}(\mathrm{Sz}, \mathrm{Sz}), \mathrm{d}(\mathrm{z}, \mathrm{z}), \mathrm{d}(\mathrm{Sz}, \mathrm{z}), \mathrm{d}(\mathrm{z}, \mathrm{Sz})\}$

$=\mathrm{d}(\mathrm{Sz}, \mathrm{z})$

Thus $\mathrm{d}(\mathrm{Sz}, \mathrm{z}) \leq \mathrm{c}(\mathrm{d}(\mathrm{Sz}, \mathrm{z}))$

If $\mathrm{Sz} \neq \mathrm{z}$ then $\mathrm{d}(\mathrm{Sz}, \mathrm{z})>0$ and hence as $\mathrm{c}$ is contractive modulus $\mathrm{c}(\mathrm{d}(\mathrm{Sz}, \mathrm{z}))<\mathrm{d}(\mathrm{Sz}, \mathrm{z})$

Therefore $\mathrm{d}(\mathrm{Sz}, \mathrm{z})<\mathrm{d}(\mathrm{Sz}, \mathrm{z})$. Which is a contradiction.

Therefore $\mathrm{Sz}=\mathrm{z}$

Hence $\mathrm{Sz}=\mathrm{Pz}=\mathrm{z}$

Now, we show that $\mathrm{z}$ is a fixed point of $\mathrm{T}$

By (ii), we have

$\mathrm{d}(\mathrm{z}, \mathrm{Tz})=\mathrm{d}(\mathrm{Sz}, \mathrm{Tz})$

Where

$$
\leq \mathrm{c}(\lambda(\mathrm{z}, \mathrm{z}))
$$

$\lambda(\mathrm{z}, \mathrm{z})=\max \{\mathrm{d}(\mathrm{Pz}, \mathrm{Qz}), \mathrm{d}(\mathrm{Pz}, \mathrm{Sz}), \mathrm{d}(\mathrm{Qz}, \mathrm{Tz}), \mathrm{d}(\mathrm{Pz}, \mathrm{Tz}), \mathrm{d}(\mathrm{Qz}, \mathrm{Sz})\}$

$=\max \{\mathrm{d}(\mathrm{z}, \mathrm{Tz}), \mathrm{d}(\mathrm{z}, \mathrm{z}), \mathrm{d}(\mathrm{Tz}, \mathrm{Tz}), \mathrm{d}(\mathrm{z}, \mathrm{Tz}), \mathrm{d}(\mathrm{Tz}, \mathrm{z})\}$

$=\mathrm{d}(\mathrm{Tz}, \mathrm{z})$

Thus $\mathrm{d}(\mathrm{z}, \mathrm{Tz}) \leq \mathrm{c}(\mathrm{d}(\mathrm{z}, \mathrm{Tz}))$

If $\mathrm{z} \neq \mathrm{Tz}$ then $\mathrm{d}(\mathrm{z}, \mathrm{Tz})>0$ and hence as $\mathrm{c}$ is contractive modulus

$\mathrm{c}(\mathrm{d}(\mathrm{z}, \mathrm{Tz}))<\mathrm{d}(\mathrm{z}, \mathrm{Tz})$

Therefore $\mathrm{d}(\mathrm{z}, \mathrm{Tz})<\mathrm{d}(\mathrm{z}, \mathrm{Tz})$. Which is a contradiction.

Hence $\mathrm{z}=\mathrm{Tz}$.

Therefore $\mathrm{Tz}=\mathrm{Qz}=\mathrm{z}$. 
Therefore $\mathrm{Sz}=\mathrm{Pz}=\mathrm{Tz}=\mathrm{Qz}=\mathrm{z}$ i.e., $\mathrm{z}$ is a common fixed point of $\mathrm{P}, \mathrm{Q}, \mathrm{S}$ and $\mathrm{T}$.

Uniqueness: For uniqueness of $\mathrm{z}$ let if possible, we assume that $\mathrm{z}$ and $\mathrm{w},(\mathrm{z} \neq \mathrm{w})$

Are common fixed point of $\mathrm{P}, \mathrm{Q}, \mathrm{S}$ and $\mathrm{T}$.

By (ii), we have

Where

$$
\begin{aligned}
\mathrm{d}(\mathrm{z}, \mathrm{w}) & =\mathrm{d}(\mathrm{Sz}, \mathrm{Tw}) \\
& \leq \mathrm{c}(\lambda(\mathrm{z}, \mathrm{w}))
\end{aligned}
$$

$(\mathrm{z}, \mathrm{w})=\max \{\mathrm{d}(\mathrm{Pz}, \mathrm{Qw}), \mathrm{d}(\mathrm{Pz}, \mathrm{Sz}), \mathrm{d}(\mathrm{Qw}, \mathrm{Tw}), \mathrm{d}(\mathrm{Pz}, \mathrm{Tw}), \mathrm{d}(\mathrm{Qw}, \mathrm{Sz})\}$

$$
\begin{aligned}
& =\max \{d(\mathrm{z}, \mathrm{w}), \mathrm{d}(\mathrm{z}, \mathrm{z}), \mathrm{d}(\mathrm{w}, \mathrm{w}), \mathrm{d}(\mathrm{z}, \mathrm{w}), \mathrm{d}(\mathrm{w}, \mathrm{z})\} \\
& =\mathrm{d}(\mathrm{z}, \mathrm{w})
\end{aligned}
$$

Thus $\mathrm{d}(\mathrm{z}, \mathrm{w}) \leq \mathrm{c}(\mathrm{d}(\mathrm{z}, \mathrm{w}))$

Since $\mathrm{z} \neq \mathrm{w}$ then $\mathrm{d}(\mathrm{z}, \mathrm{w})>0$ and hence as $\mathrm{c}$ is contractive modulus $\mathrm{c}(\mathrm{d}(\mathrm{z}, \mathrm{w}))<\mathrm{d}(\mathrm{z}, \mathrm{w})$.

Therefore $\mathrm{d}(\mathrm{z}, \mathrm{w})<\mathrm{d}(\mathrm{z}, \mathrm{w})$, which is a contradiction.

Therefore $\mathrm{z}=\mathrm{w}$.

Thus $\mathrm{z}$ is the unique common fixed point of $\mathrm{P}, \mathrm{Q}, \mathrm{S}$ and $\mathrm{T}$.

Hence the theorem.

Corollary 3.2 Let (X, d) be a complete metric space. Suppose that the mapping P, S and

$\mathrm{T}$ are self-maps of $\mathrm{X}$ satisfying the following conditions:

1. $\mathrm{T}(\mathrm{X}) \subseteq \mathrm{P}(\mathrm{X})$ and $\mathrm{S}(\mathrm{X}) \subseteq \mathrm{P}(\mathrm{X})$;

2. $\mathrm{d}(\mathrm{Sx}, \mathrm{Ty}) \leq \mathrm{c}(\lambda(\mathrm{x}, \mathrm{y}))$

Where $c$ is an upper semi continuous, contractive modulus and

$\lambda(\mathrm{x}, \mathrm{y})=\max \{\mathrm{d}(\mathrm{Px}, \mathrm{Py}), \mathrm{d}(\mathrm{Px}, \mathrm{Sx}), \mathrm{d}(\mathrm{Py}, \mathrm{Ty}), \mathrm{d}(\mathrm{Px}, \mathrm{Ty}), \mathrm{d}(\mathrm{Py}, \mathrm{Sx})\}$

3. The pair (S, P) and (T, P) are weakly compatible.

Then $\mathrm{P}, \mathrm{S}$ and $\mathrm{T}$ have a unique common fixed point.

proof: $\mathrm{By}$ taking $\mathrm{P}=\mathrm{Q}$ in theorem 3.1 we get the proof.

Corollary 3.3 Let $(X, d)$ be a complete metric space. Suppose that the mappings $P$ and S are self-maps of X satisfying the following conditions:

(1) $\mathrm{S}(\mathrm{X}) \subseteq \mathrm{P}(\mathrm{X})$

(2) $d(\operatorname{Sx}, S y)<c(\lambda(x, y))$

Where $\mathrm{c}$ is an upper semi continuous, contractive modulus and

$\lambda(x, y)=\max \{d(P x, P y), d(P x, S x), d(P y, S y), d(P x, S y), d(P y, S x)$

(3) the pair $(\mathrm{S}, \mathrm{P})$ is weakly compatible

Then $P$ and $S$ have a unique common fixed point.

Proof: By taking $\mathrm{P}=\mathrm{Q}$ and $\mathrm{S}=\mathrm{T}$ in theorem 3.1 we get the proof.

\section{Acknowledgements}

The authors are grateful to the referees for careful readings and corrections. The authors are also grateful to Dr. A. K. Gupta for his cooperation and valuable suggestions in the preparation of this paper.

\section{References}

[1] Aamri, M. and El Moutawakil, D.: some new common fixed theorems under strict conditions, J. Math. Anal. Appl., 270(2002), 181188.

[2] Fisher, B.: Common Fixed Point of Four Mapping. Bull. Inst. Of Math. Academia. Sinicia. 11(1983), 103-113.

[3] Imdad, M. Kumar, Santosh. And Khan, M. S.:Remarks on fixed Point theorems satisfying implicit relations, Radovi Math., 11(2002), 135-143.

[4] Jungck, G.: Compatible mappings and common fixed points, internet. I. Math and Math. Sci., 9(1986), 771-779.

[5] Jungck, G.and Rhoades, B.E.: Fixed Point for set valued Functions Without Continuty, Indian J. Pure Appl. Math, 29(1998), 227238.

[6] Motkowski, J.: Fixed point theorem for mappings with a contractive iterate at a point, Amer. Math. Soc., 62(1977),344-348.

[7] Pant, R.P.: Common fixed point of contractive maps. J. math. And Appl., 226(1998), 251-258.

[8] Sessa,S.: On a weak commutativity condition of mappings in a fixed point considerations, publ. inst math. Debre, 32(1982), 149153. 